\title{
Extracting token-level signals of syntactic processing from fMRI - with an application to PoS induction
}

\author{
Joachim Bingel Maria Barrett Anders Søgaard \\ Centre for Language Technology, University of Copenhagen \\ Njalsgade 140, 2300 Copenhagen S, Denmark \\ \{bingel, barrett, soegaard\}@hum.ku.dk
}

\begin{abstract}
Neuro-imaging studies on reading different parts of speech (PoS) report somewhat mixed results, yet some of them indicate different activations with different PoS. This paper addresses the difficulty of using fMRI to discriminate between linguistic tokens in reading of running text because of low temporal resolution. We show that once we solve this problem, fMRI data contains a signal of PoS distinctions to the extent that it improves PoS induction with error reductions of more than $4 \%$.
\end{abstract}

\section{Introduction}

A few recent studies have tried to extract morphosyntactic signals from measurements of human sentence processing and used this information to improve NLP models. Klerke et al. (2016), for example, used eye-tracking recordings to regularize a sentence compression model. More related to this work, Barrett et al. (2016) recently used eyetracking recordings to induce PoS models. However, a weakness of eye-tracking data is that while eye movement surely does reflect the temporal aspect of cognitive processing, it is only a proxy of the latter and does not directly represent which processes take place in the brain.

A recent neuro-imaging study suggests that concrete nouns and verbs elicit different brain signatures in the frontocentral cortex, and that concrete and abstract nouns elicit different brain activation patterns (Moseley and Pulvermüller, 2014). Also, for example, concrete verbs activate motor and premotor cortex more strongly than concrete nouns, and concrete nouns activate inferior frontal areas more strongly than concrete verbs. A decade earlier, Tyler et al. (2004) showed that the left inferior frontal gyrus was more strongly activated in processing regularly inflected verbs compared to regularly inflected nouns.

Such studies suggest that different parts of our brains are activated when reading different parts of speech (PoS). This would in turn mean that neuro-images of readers carry information about the grammatical structure of what they read. In other words, neuro-imaging provides a partial, noisy annotation of the data with respect to morphosyntactic category.

Say neuro-imaging data of readers was readily available. Would it be of any use to, for example, engineers interested in PoS taggers for lowresource languages? This is far from obvious. In fact, it is well-known that neuro-imaging data from reading is noisy, in part because the reading signal is not always very distinguishable (Tagamets et al., 2000), and also because the content of what we read may elicit certain activation in brain regions e.g. related to sensory processing (Boulenger et al., 2006; González et al., 2006).

Other researchers such as Borowsky et al. (2013) have also questioned that there are differences, claiming to show that the majority of activation is shared between nouns and verbs - including in regions suggested by previous researchers as unique to either nouns or verbs. Berlingeri et al. (2008) argue that only verbs could be associated with unique regions, not nouns.

In this paper we nevertheless explore this question. The paper should be seen as a proof of concept that interesting linguistic signals can be extracted from brain imaging data, and an attempt to show that learning NLP models from such data could be a way of pushing the boundaries of both fields.

Contributions (a) We present a novel technique for extracting syntactic processing signal at the token level from neuro-imaging data that is charac- 

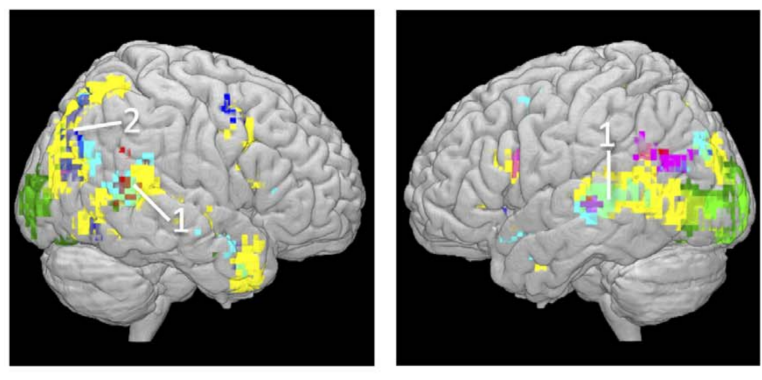

Characters

Visual

\section{Syntax}

Dialog
Semantics

Motion
Figure 1: Neural activity by brain region and type of information processed, as measured and rendered by Wehbe et al. (2014).

terized by low temporal resolution. (b) We demonstrate that the fMRI data improves performance of a type-constrained, second order hidden Markov model for PoS induction. Our model leads to an error reduction of more than $4 \%$ in tagging accuracy despite very little training data, which to the best of our knowledge is the first positive result on weakly supervised part-of-speech induction from fMRI data in the literature.

\section{2 fMRI}

Functional Magnetic Resonance Imaging (fMRI) is a technology for spatial visualization of brain activity. It measures the changes in oxygenation of the blood in the brain, often by use of the blood oxygenation level-dependent contrast (Ogawa et al., 1992), which correlates with neural activity. While the spatial resolution of fMRI is very high, its temporal resolution is low compared to other brain imaging technologies like EEG, which usually returns millisecond records of brain activity, but on the contrary have low spatial resolution. The temporal resolution of fMRI is usually between $0.5 \mathrm{~Hz}$ and $1 \mathrm{~Hz}$. fMRI data contains representations of neural activity of millimeter-sized cubes called voxels.

The high spatial resolution may enable us to detect fine differences in brain activation patterns, such as between processing nouns and verbs, but the low temporal resolution is a real challenge when the different tokens are processed serially and quickly after each other, as is the case in reading.

Another inherent challenge when working with
fMRI data is the lag between the the reaction to a stimulus and the point when it becomes visible through fMRI. This lag is called the hemodynamic response latency. While we know from brain imaging technologies with higher temporal resolution that the neural response to a stimuli happens within milliseconds, it only shows in fMRI data after a certain period of time, which further blurs the low temporal dimension of serial fMRI recordings. This latency has been studied as long as fMRI technology itself. It depends on the blood vessels and varies between e.g. voxels, brain regions, subjects, and tasks. A meta study of the hemodynamic response report latencies between 4 and 14 seconds in healthy adults, though latencies above 11 seconds are less typically reported (Handwerker et al., 2012). According to Handwerker et al. (2012), the precise response shape for a given stimulus and voxel region is hard to predict and remains a challenge when modeling temporal aspects of fMRI data.

Figure 1 visualizes the neural activations in different brain regions as a reaction to the type of information that is processed during reading. See Price (2012) for a thorough review of fMRI language studies.

Wehbe et al. (2014) presented a novel approach to fMRI studies of linguistic processing by studying a more naturalistic reading scenario, and modeling the entire process of reading and story understanding. They used data from 8 subjects reading contextualized, running text: a chapter from a Harry Potter book. The central benefit of this approach is that it allows studies of complex text processing closer to a real-life reading experience. Wehbe et al. (2014) used this data to train a comprehensive, generative model that-given a text passage — could predict the fMRI-recorded activity during the reading of this passage. Using the same data, our goal is to model a specific aspect of the story understanding process, i.e. the grammatical processing of words.

\section{Data}

\subsection{Textual data}

We use the available fMRI recordings from Wehbe et al. (2014), where 8 adult, native English speakers read chapter 9 from Harry Potter and the Sorcerer's Stone in English. The textual data as provided in the data set does not explicitly mark sentence boundaries, neither is punctuation sep- 


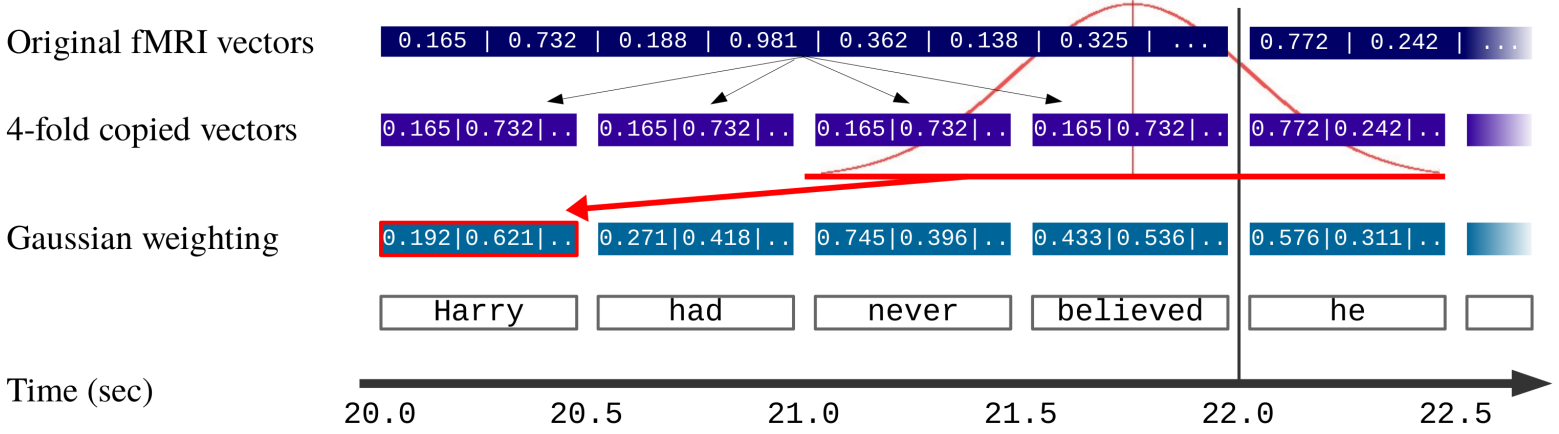

Figure 2: Computation of token-level fMRI vectors from the original fMRI data for the first token "Harry" while accounting for hemodynamic response latency using a Gaussian sliding window over a certain time window (indicated by red horizontal line). The final fMRI vector for "Harry" (red box) is computed as specified in Equation 1. In this example, the time stamp $t$ for the token is $20 s$ and the time window stretches from $t+1 s$ to $t+2.5 s$.

arated from the tokens at the end of clauses and sentences. As the temporal alignment between tokens and fMRI recordings (see below) forbids us to detach punctuation marks from their preceding tokens and introduce them as new tokens, we opt to remove all punctuation from the data. In the same process, we use simple heuristics to detect sentence boundaries. Finally, we correct errors in sentence splitting manually.

The chapter counts 4,898 tokens (excluding punctuation) and 1,411 types in 408 sentences.

\section{2 fMRI data}

The fMRI data from the same data set is available as high-dimensional vectors of flattened thirdorder tensors, in which each component represents the blood-oxygen-level dependent contrast for a certain voxel in the three-dimensional fMRI image. The resolution of the image is at $3 \times 3 \times 3 \mathrm{~mm}$, such that the brain activity for the eight subjects is represented by approximately 31,400 voxels on average (standard deviation is 3,607 ) depending on the size of their brain.

This data is recorded every two seconds during the reading process, in which each token is consecutively displayed for 0.5 seconds on a screen inside the fMRI scanner. Prior to reading, the subjects are asked to focus on a cross displayed at the center of the screen in a warm-up phase of 20 seconds. The chapter is divided into four blocks, separated by additional concentration phases of 20 seconds. Furthermore, paragraphs are separated by a 0.5 -seconds display of a cross at the center of the screen.
As mentioned in the preceding section, punctuation marks were not displayed separately, but instead attached to the preceding token. This is arguably motivated through the attempt to create a reading scenario that is as natural as possible within the limitations of an fMRI recording. In similar fashion, contractions such as don't or he's were represented as one token, just as they appear in the original text.

In order to make the data feasible for our HMM approach (see Section 4), we apply Principal Component Analysis (PCA) to the high-dimensional fMRI vectors. We initially tune the number of principal components, which we describe in Section 5.

\subsubsection{Computing token-level fMRI vectors}

As outlined above, the time resolution of the fMRI recordings means that every block of four consecutive tokens is time-aligned with a single fMRI image. Naturally, this shared representation of consecutive tokens complicates any language learning at the token level. Furthermore, the hemodynamic response latency inherent to fMRI recordings entails that the image recorded while reading a certain token most probably does not give any clues about the mental state elicited by this stimulus.

We therefore face the dual challenge of

1. inferring token-level information from supratoken recordings, and

2. identifying the lag after which the perceptual effects of reading a given token are visible. 


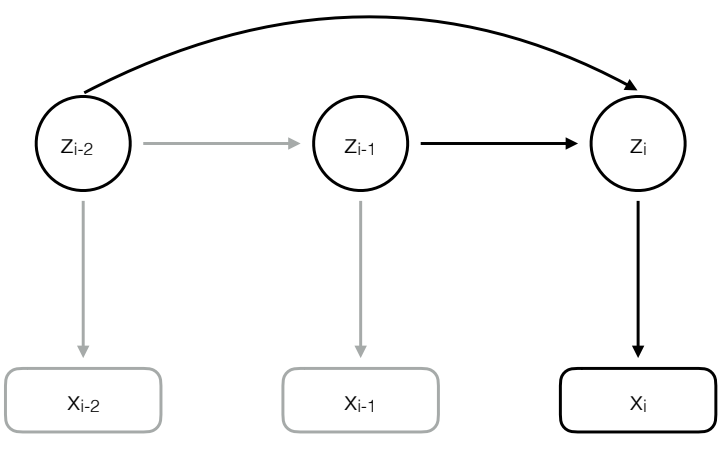

Figure 3: Second-order HMM incorporating transitional probabilities from first and second-degree preceding states.

We address this problem through the following procedure that we illustrate in Figure 2. First, we copy the number of fMRI recordings fourfold, such that every fMRI vector is aligned to exactly one token (excluding the vectors that are recorded while no token was displayed). The representation for a given token is then computed as a weighted average over all fMRI vectors that lie within a certain time window in relation to the token in question. Two consecutive tokens that originally lie within the same block of four thus receive different representations, provided that the window is large enough to transcend the border between two blocks.

The fMRI representation for the token at time stamp $t$ is given by

$$
v_{t}=\frac{1}{|V|} \sum_{k=1}^{|V|} V_{k} \cdot w_{k}
$$

where $V$ is the series of fMRI vectors within the time window $[t+s, t+e]$, and $w$ is a Gaussian window of $|V|$ points, with a standard deviation of 1. In factoring the Gaussian weight vector into the equation, we lend less weight to the fMRI recordings at the outset and at the end of the time window specified through $s$ (start) and $e$ (end).

\section{Model}

We use a second-order hidden Markov model (HMM) with Wiktionary-derived type constraints (Li et al., 2012) as our baseline for weakly supervised PoS induction. We use the original implementation by Li et al. (2012). The model is a type-constrained, second order version of the first-order featurized HMM previously introduced by Berg-Kirkpatrick et al. (2010).

In each state $z_{i}$, a PoS HMM generates a sequence of words by consecutively generating word emissions $x_{i}$ and successor states $z_{i+1}$. The emission probabilities and state transition probabilities are multinomial distributions over words and PoS. The joint probability of a word sequence and a tag sequence is

$$
P_{\theta}(x, z)=P_{\theta}\left(z_{1}\right) \prod_{i=1} P_{\theta}\left(x_{i} \mid z_{i}\right) \prod_{i=2} P_{\theta}\left(z_{i} \mid z_{i-1}\right)
$$

Following Berg-Kirkpatrick et al. (2010), the model calculates the probability distribution $\theta$ that parameterizes the emission probabilities as the output of a maximum entropy model, which enables unsupervised learning with a rich set of features. We thus let

$$
\theta_{x_{i}, z_{i}}=\frac{\exp \left(\mathbf{w}^{\top} \mathbf{f}\left(x_{i}, z_{i}\right)\right)}{\sum_{x^{\prime}} \exp \left(\mathbf{w}^{\top} \mathbf{f}\left(x^{\prime}, z_{i}\right)\right)}
$$

where $\mathbf{w}$ is a weight vector and $\mathbf{f}\left(x_{i}, z_{i}\right)$ is a feature function that will, in our case, consider the fMRI vectors $v_{t}$ that we computed in section 3.2.1 and a number of basic features that we adopt from the original model (Li et al., 2012). See Section 5 for details.

In addition, we use a second-order HMM, first introduced for PoS tagging in Thede and Harper (1999), in which transitional probabilities are also considered for second-degree subsequent states (cf. figure 3). Here, the joint probability becomes

$$
\begin{array}{r}
P_{\theta}(x, z)=P_{\theta}\left(z_{1}\right) P_{\theta}\left(x_{1} \mid z_{1}\right) P_{\theta}\left(z_{2} \mid z_{1}\right) \\
\prod_{i=2} P_{\theta}\left(x_{i} \mid z_{i}\right) \prod_{i=3} P_{\theta}\left(z_{i} \mid z_{i-2}, z_{i-1}\right)
\end{array}
$$

In order to optimize the HMM (including the weight vector w), the model uses the EM algorithm as applied for feature-rich, locally normalized models introduced in Berg-Kirkpatrick et al. (2010), with the important modification that we use type constraints in the E-step, following $\mathrm{Li}$ et al. (2012). Specifically, for each state $z_{i}$, the emission probability $P\left(x_{i} \mid z_{i}\right)$ is initialized randomly for every word type associated with $z_{i}$ in our tag dictionary (the type constraints). This weakly supervised setup allows us to predict the actual PoS tags instead of abstract states. The Mstep is solved using L-BFGS (Liu and Nocedal, 1989) 


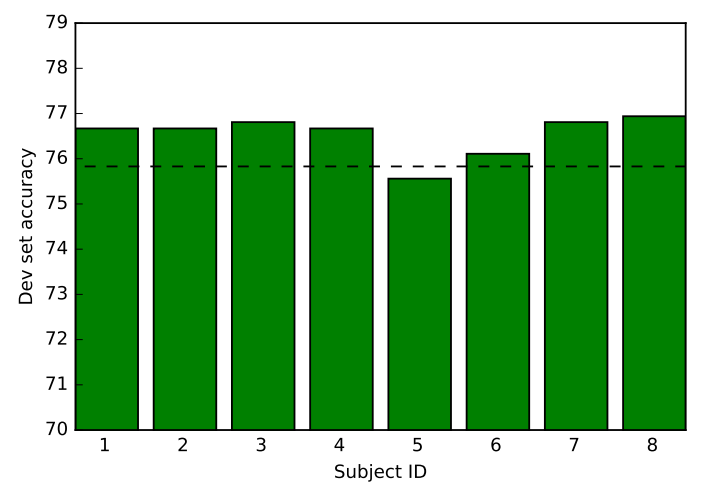

Figure 4: Accuracy on the development set for the different subjects when trained and tested on fMRI data from only this one subject. Dashed line is the development set baseline. Only in one out of eight cases does adding fMRI features lead to worse performance.

EM-HMM Parameters We use the same setting as Li et al. (2012) for the number of EM iterations, fixing this parameter to 30 for all experiments.

\section{Experiments}

Experimental setup From the neuro-imaging dataset described above, we use 41 sentences ( 720 tokens) as a development set and 41 sentences (529 tokens) as a test set, and the remaining 326 sentences (corresponding to 80\%) for training our model.

Basic features The basic features of all the models (except when explicitly stated otherwise) are based on seven features that we adopt from $\mathrm{Li}$ et al. (2012), capturing word form, hyphenation, suffix patterns, capitalization and digits in the token.

Wiktionary Of the 1,411 word types in the corpus, we find that $1,381(97.84 \%)$ are covered by the Wiktionary dump made available by Li et al. (2012), ${ }^{1}$ which we use as our type constraints when inducing our models.

\subsection{Part-of-speech annotation}

Though Wehbe et al. (2014) also provide syntactic information, these are automatic parses that are not suitable for the evaluation of our model. The development and test data are therefore manually

\footnotetext{
1https://code.google.com/archive/p/ wikily-supervised-pos-tagger/
}

annotated for universal part-of-speech tags (Petrov et al., 2011) by two linguistically trained annotators. The development set was annotated by both annotators, who reached an inter-annotator agreement of 0.926 in accuracy and 0.928 in weighted $F_{1}$. For the final development and test data, disagreements were resolved by the annotators.

\subsection{Non-fMRI baselines}

Our first baseline is a second-order HMM with type constraints from Wiktionary; this in all respects the model proposed by Liu et al. (2012), except trained on our small Harry Potter corpus. In a second baseline model, we also incorporate 300dimensional GloVe word embeddings trained on Wikipedia and the Gigaword corpus (Pennington et al., 2014). We also test a version of the baseline without the basic features to get an estimate of the contribution of this aspect of the setup.

\subsection{Token-level fMRI}

We run a series of experiments with token-level fMRI vectors that we obtain as described in Section 3.2.1. Initially, we train separate models for each of the eight individual subjects, whose performance on the development data are illustrated in Figure 4.

\subsubsection{Tuning hyperparameters}

We tune the following hyperparameters on the token-level development set in the following order: the number of subjects to use, the number of principal components per subject, and the time window. For the earlier tuning processes we fix the later hyperparameters to values we consider reasonable, but once we have tuned a hyperparameter, we use the best value from this tuning process for later tuning steps. The initial values are: 10 principal components and a time window of $[t+0 s$, $t+6 s]$.

Number of subjects To reduce the chance of overfitting, we use fMRI data from several readers in our model. The data from Wehbe et al. (2014) would in theory allow us to average the three-dimensional image space for any number of readers, but this is not feasible if only for the difference in brain sizes between the subjects. It is not feasible, either, to average over the eigenvectors that we obtain from PCA, as the eigenvectors between subjects do not share the same (or any concrete) feature space. We therefore concatenate 


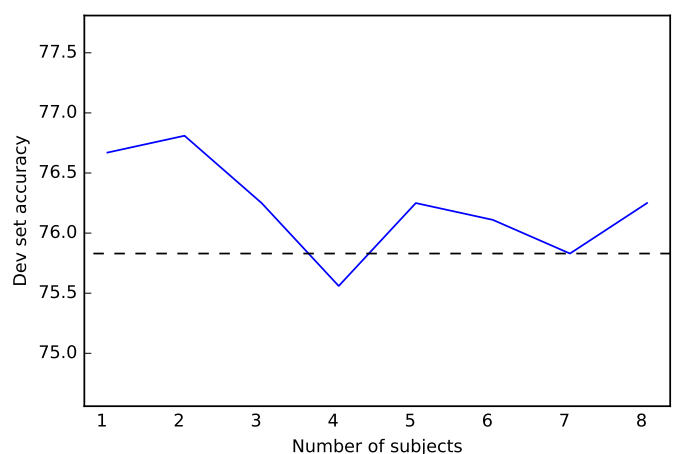

(a) Learning curve for increasing number of subjects in the model. Fixed hyper-parameters: 10 principal components and a time window of $[t+0 s, t+6 s]$.

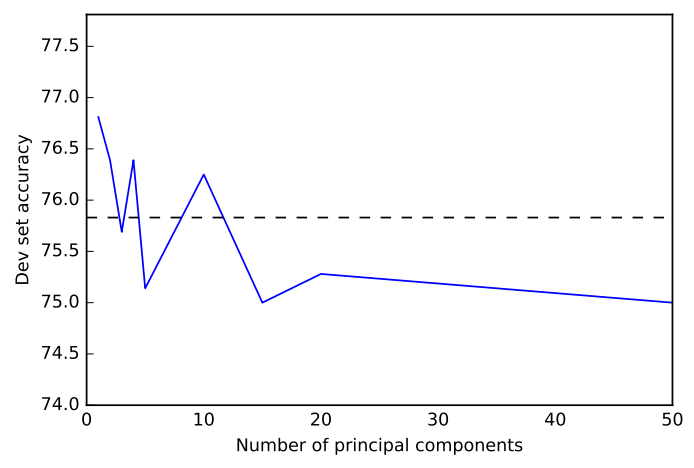

(b) Learning curve for increasing number of principal components per subject in the model. Number of principal components $\in\{1,2,3,4,5,10,15,20,50\}$ Fixed hyperparameters: 8 subjects, a time window of $[t+0 s, t+6 s]$.

Figure 5: Exploring two individual hyper-parameters of the model on development set. Dashed lines indicate the development set baseline.

the eigenvectors that we obtain for different subjects, such that the feature vectors grow in length as the number of included subjects increases.

As Figure 5a shows, exploring an increasing number of subjects in the model does not seem to a have consistent effect on development set accuracy. However, we expect an increased robustness from a model that incorporates a greater number of subjects. In all following experiments we therefore use data from all eight readers, but we would also expect a model with fewer subjects to perform reasonably.

Principal components Fixing the number of subjects to eight, we then perform experiments to determine the number of principal components per subject to consider in our model, whose results are visualized in Figure 5b. We observe the first eigenvectors carry a strong signal, while a great number of principal components tends to water down the signal and lead to worse performance. We choose to continue using 10 dimensions in all further experiments.

Time window for token vectors We next run experiments to determine the optimal time window for the computation of the token vectors, using different combinations of start and end times in relation to the token time stamps, but keeping the number of subjects and principal components constant at eight and ten, respectively. These experiments yield three different time windows with an equally good performance on the development set: $[t-4 s, t+10 s],[t+2 s, t+8 s]$ and $[t+0 s$, $t+6 s]$. Note that due to the Gaussian weighting the centre of the interval gets more weight than the edges and that $[t-4 s, t+10 s]$ and $[t+0 s, t+6 s]$ have the same centre, $t+3$. While $[t+2 s, t+8 s]$ and $[t+0, t+6]$ align better with psycholinguistic expectations, $[t-4 s, t+10 s]$ makes our model less prone to overfitting. We therefore select the model averaging over the largest time window.

\subsection{Type-level fMRI aggregates}

Next, we aggregate token vectors to compute their type-level averages, in an effort to explore to which degree neural activity is dependent on the read word type rather than the concrete grammatical environment, and whether this can allow our model to draw conclusions about the grammatical class of a token. We compute the type-level aggregates as the component-wise arithmetic mean of the token vectors that we extract using the parameter settings optimized above. Note, however, that out of the 4,898 tokens in the text, 823 (16.9\%) occur only once.

\section{Results}

Table 1 reports the results that we obtain with our final hyper-parameter settings, which are as follows:

$\begin{array}{ll}\text { Number of subjects } & 8 \\ \text { Principal components } & 10 \\ \text { Start of time window } & t-4 s \\ \text { End of time window } & t+10 s\end{array}$

The results show that our model leads to a consid- 


\begin{tabular}{lr}
\hline & Accuracy \\
\hline Baseline (Li et al., 2012) & 69.57 \\
\hline Baseline+GloVe & 69.38 \\
Baseline w/o basic feats & 55.53 \\
\hline fMRI (token-level) w/o basic feats & 56.99 \\
fMRI (type-level) & 70.32 \\
fMRI (token-level) & $\mathbf{7 0 . 8 9}$ \\
\hline Error reduction over baseline & 04.34 \\
\hline
\end{tabular}

Table 1: Tagging accuracy on test data for the different models. The fMRI model is significantly better than the baseline ( $p=0.014$, Bootstrap).

\begin{tabular}{lrrrr}
\hline Class & Prec. & Rec. & $F_{1}$ & \pm BL \\
\hline ADJ & 37.50 & 42.86 & 40.00 & +2.71 \\
ADP & 83.67 & 77.36 & 80.39 & +1.54 \\
ADV & 66.00 & 58.93 & 62.26 & +5.69 \\
CONJ & 70.97 & 70.97 & 70.97 & \pm 0.00 \\
DET & 80.49 & 80.49 & 80.49 & +3.38 \\
NOUN & 70.37 & 76.00 & 73.08 & +0.28 \\
NUM & 00.00 & 00.00 & 00.00 & -20.00 \\
PRON & 88.68 & 74.60 & 81.03 & +4.76 \\
PRT & 41.67 & 41.67 & 41.67 & +11.67 \\
VERB & 74.36 & 76.32 & 75.32 & -0.95 \\
\hline
\end{tabular}

Table 2: Test data tagging performance by part-ofspeech class for the best fMRI model. The rightmost column displays the difference in $F_{1}$ compared to the baseline model.

erable error reduction over the baseline model as well as the embeddings-enriched baseline model. It also outperforms the model which uses typelevel averages over the fMRI recordings. Leaving out the basic features hurts performance, but even without the basic features the fMRI data can reduce error with $3.28 \%$ on the test set. In Table 2 we present the performance on the individual PoS classes under our best model.

\section{Analysis and Discussion}

\subsection{What's in the fMRI vectors?}

t-SNE (Van der Maaten and Hinton, 2008) is a powerful supervised dimensionality reduction tool for visualizing high-dimensional data in twodimensional space using Stochastic Neighbor Embedding. In Figure 6, we visualize pairs of PoS classes of the test data in a two-dimensional re-

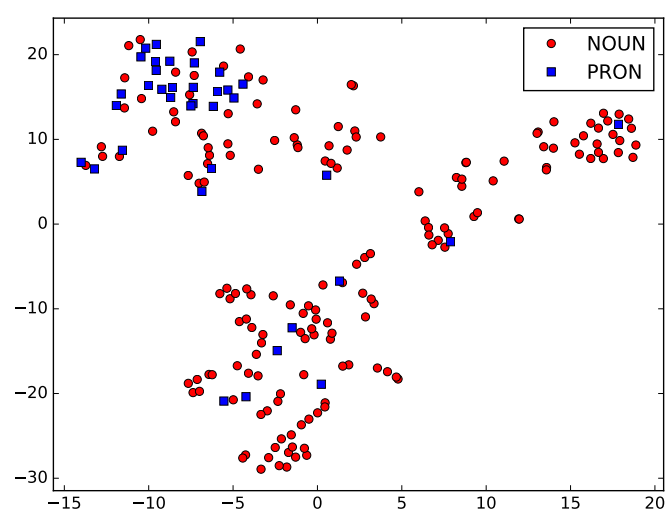

(a) NOUN and PRON

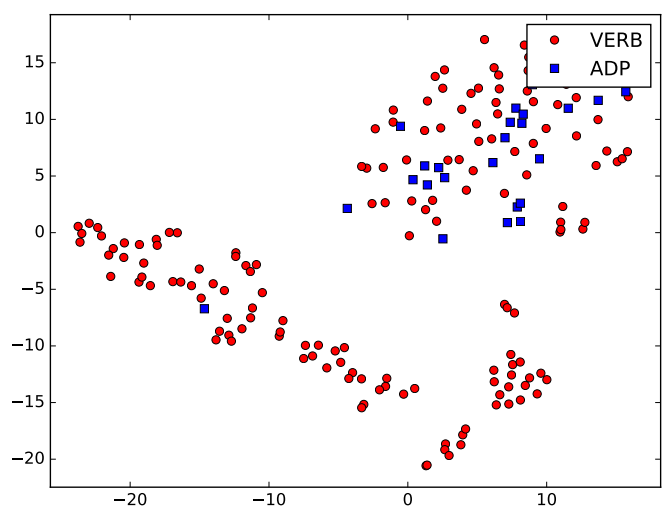

(b) VERB and ADP

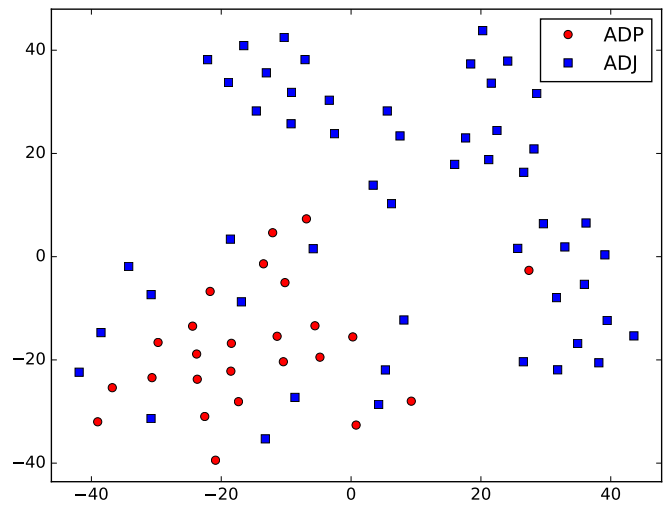

(c) ADP and ADJ

Figure 6: Selected t-SNE visualizations of fMRI vectors for all tokens of a class of the test set. The visualizations show that datapoints of a PoS class tend to cluster in the fMRI vector space. 
duction of the embedding space obtained when using the settings of the best fMRI model. The fact that we can discriminate reasonably well between, e.g., nouns and pronouns, verbs and adpositions, as well as adpositions and adjectives on the basis of fMRI data is to the best of our knowledge a new finding.

\subsection{Discussion of the results}

We showed that by careful model tuning and design it is possible to extract a signal of grammatical processing in the brain from fMRI. The figures that we present in Table 1 reflect, to our knowledge, the first successful results in inferring grammatical function at the token level from fMRI data. Our best model, which we train on the ten principal components from the fMRI recordings of eight readers, achieves an error reduction of over $4 \%$ despite a very small amount of training data. We find that our best model uses a very wide window of fMRI recordings to compute the representations for individual tokens, considering all recordings from 4 seconds before the token is displayed until 10 seconds after the token is displayed. Our best explanation for why the incorporation of preceding fMRI measurements is beneficial to our model, is that the grammatical function of a token may be predictable from a reader's cognitive state while reading preceding tokens. However, note that the measurements at the far ends of the time window only factor into the token vector to a small degree as a consequence of the Gaussian weighting. Our experiments further suggest that using token-level information instead of type-level features, such as word embeddings or type averages of fMRI vectors, is helpful for PoS induction that already is type-constrained.

Recently, Huth et al. (2016) found that semantically related words are processed in the same area of the brain. Open questions for future work include whether there is a bigger potential for using fMRI data for semantic rather than syntactic NLP tasks, and whether the signal we find mainly stems from semantic processing differences.

\section{Conclusion}

This paper presents the first experiments inducing part of speech from fMRI reading data. Cognitive psychologists have debated whether grammatical differences lead to different brain activation patterns. Somewhat surprisingly, we find that

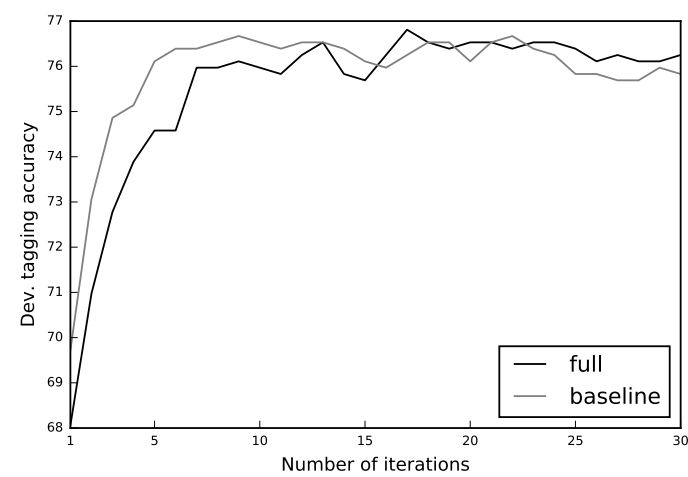

Figure 7: Learning curve of tagging accuracy on the development set as a function of different number of EM iterations for baseline model and the full model for iteration numbers $\in[1,30]$. Fixed hyper-parameters: 8 subjects, 10 principal components, and a time window of $t-4 \mathrm{~s}$ to $t+10 \mathrm{~s}$

the fMRI data contains a strong signal, enabling a $4 \%$ error reduction over a state-of-the-art unsupervised PoS tagger. While our approach may not be readily applicable for developing NLP models today, we believe that the presented results may inspire NLP researchers to consider learning models from combinations of linguistic resources and auxiliary, behavioral data that reflects human cognition.

\section{Acknowledgements}

This research was partially funded by the ERC Starting Grant LOWLANDS No. 313695, as well as by Trygfonden.

\section{Supplementary material}

Number of EM iterations As supplementary material, we present the EM learning curve in Figure 7, which shows a steep learning curve at the beginning and relatively stable performance figures after 15 iterations for the full model and 10 iterations for the baseline model.

\section{References}

Maria Barrett, Joachim Bingel, Frank Keller, and Anders Søgaard. 2016. Weakly supervised part-ofspeech induction using eye-tracking data. In $A C L$.

Taylor Berg-Kirkpatrick, Alexandre Bouchard-Cote, John DeNero, and Dan Klein. 2010. Painless unsupervised learning with features. In Proceedings of NAACL, pages 582-590. 
Manuela Berlingeri, Davide Crepaldi, Rossella Roberti, Giuseppe Scialfa, Claudio Luzzatti, and Eraldo Paulesu. 2008. Nouns and verbs in the brain: Grammatical class and task specific effects as revealed by fMRI. Cognitive Neuropsychology, 25(4):528-558.

Ron Borowsky, Carrie Esopenko, Layla Gould, Naila Kuhlmann, Gordon Sarty, and Jacqueline Cummine. 2013. Localisation of function for noun and verb reading: converging evidence for shared processing from fmri activation and reaction time. Language and Cognitive Processes, 28(6):789-809.

Véronique Boulenger, Alice C Roy, Yves Paulignan, Viviane Deprez, Marc Jeannerod, and Tatjana A Nazir. 2006. Cross-talk between language processes and overt motor behavior in the first $200 \mathrm{msec}$ of processing. Journal of cognitive neuroscience, 18(10):1607-1615.

Julio González, Alfonso Barros-Loscertales, Friedemann Pulvermüller, Vanessa Meseguer, Ana Sanjuán, Vicente Belloch, and César Ávila. 2006. Reading cinnamon activates olfactory brain regions. Neuroimage, 32(2):906-912.

Daniel A Handwerker, Javier Gonzalez-Castillo, Mark D'Esposito, and Peter A Bandettini. 2012. The continuing challenge of understanding and modeling hemodynamic variation in fmri. Neuroimage, 62(2):1017-1023.

Alexander G Huth, Wendy A de Heer, Thomas L Griffiths, Frédéric E Theunissen, and Jack L Gallant. 2016. Natural speech reveals the semantic maps that tile human cerebral cortex. Nature, 532(7600):453458.

Sigrid Klerke, Yoav Goldberg, and Anders Søgaard. 2016. Improving sentence compression by learning to predict gaze. In NAACL.

Shen Li, João Graça, and Ben Taskar. 2012. Wikily supervised part-of-speech tagging. In EMNLP, pages 1389-1398.

Dong C Liu and Jorge Nocedal. 1989. On the limited memory bfgs method for large scale optimization. Mathematical programming, 45(1-3):503-528.

Xiaohua Liu, Ming Zhou, Furu Wei, Zhongyang Fu, and Xiangyang Zhou. 2012. Joint inference of named entity recognition and normalization. In $A C L$, pages 526-535.

Rachel L Moseley and Friedemann Pulvermüller. 2014. Nouns, verbs, objects, actions, and abstractions: local fmri activity indexes semantics, not lexical categories. Brain and language, 132:28-42.

Seiji Ogawa, David W Tank, Ravi Menon, Jutta M Ellermann, Seong G Kim, Helmut Merkle, and Kamil Ugurbil. 1992. Intrinsic signal changes accompanying sensory stimulation: functional brain mapping with magnetic resonance imaging. Proceedings of the National Academy of Sciences, 89(13):5951-5955.

Jeffrey Pennington, Richard Socher, and Christopher D Manning. 2014. Glove: Global vectors for word representation. In EMNLP, volume 14, pages 15321543.

Slav Petrov, Dipanjan Das, and Ryan McDonald. 2011. A universal part-of-speech tagset. CoRR abs/1104.2086.

Cathy J Price. 2012. A review and synthesis of the first 20 years of pet and fmri studies of heard speech, spoken language and reading. Neuroimage, 62(2):816847.

M-A Tagamets, Jared M Novick, Maria L Chalmers, and Rhonda B Friedman. 2000. A parametric approach to orthographic processing in the brain: an fmri study. Cognitive Neuroscience, Journal of, 12(2):281-297.

Scott Thede and Mary Harper. 1999. A second-order hidden markov model for part-of-speech tagging. In $A C L$, pages 175-182.

Lorraine Tyler, Peter Bright, Paul Fletcher, and Emmanuel Stamatakis. 2004. Neural processing of nouns and verbs: The role of inflectional morphology. Neuropsychologia, 42(4):512-523.

Laurens Van der Maaten and Geoffrey Hinton. 2008. Visualizing data using t-sne. Journal of Machine Learning Research, 9(2579-2605):85.

Leila Wehbe, Brian Murphy, Partha Talukdar, Alona Fyshe, Aaditya Ramdas, and Tom Mitchell. 2014. Simultaneously uncovering the patterns of brain regions involved in different story reading subprocesses. PloS one, 9(11):e112575. 